\title{
Revista Brasileira de Enfermagem REBEn \\ Nova definição de casos de Sífilis Congênita para fins de vigilância epidemiológica no Brasil, 2004
}

New case definition of Congenital Syphilis

for epidemiological surveillance purposes in Brazil, 2004

Nueva definición de casos de Sifilis Congénita

para finalidad de vigilancia epidemiologica en Brasil, 2004

Leidijany Costa Paz

Técnica da Unidade de Epidemiologia do Programa Nacional de DST/AIDS do Ministério da Saúde do Brasil.

Gerson Fernando Pereira

Técnico da Unidade de Epidemiologia do Programa Nacional de DTS/AIDS do Ministério da Saúde do Brasil.

Valdir Monteiro Pinto

Assessor responsável pela Unidade de Doenças Sexualmente Transmissíveis do Programa Nacional de DST/AIDS do Ministério da Saúde do Brasil.

\section{Maria Goretti Pereira Fonseca Medeiros}

Assessora responsável pela Unidade de Epidemiologia do Programa Nacional de DST/ AIDS do Ministério da Saúde do Brasil.

Luiza Harunari Matida

Técnica da Coordenação Estadual de São Paulo, Secretaria de Estado da Saúde de São Paulo. Responsável pelo Grupo de Transmissão Vertical do HIV e Outras Infecções Congênitas. Integrante do Comitê Gestor de Epidemiologia do Programa Nacional de DST/AIDS do Ministério da Saúde do

Brasil.

Valéria Saraceni

Coordenação de Doenças Transmissíveis Secretaria Municipal de Saúde do Rio de Janeiro. Integrante do Comitê Assessor para as Recomendações de terapia Anti-Retroviral em Gestantes e Profilaxia da Transmissão Vertical do

HIV.

Alberto Novaes Ramos Jr

Professor do Departamento de Saúde Comunitária da Universidade Federal do Ceará. Integrante do Comitê Assessor de Epidemiologia do Programa Nacional de DST/AIDS do Ministério da Saúde do Brasil.
As ações de vigilância epidemiológica de qualquer agravo ou doença demandam estratégias bem definidas para a identificação de eventos relacionados ao processo saúde-doença que requeiram ações de saúde pública associadas ao planejamento, ao monitoramento e à avaliação de programas $^{(1)}$.

A definição de caso em epidemiologia constitui-se em uma dessas estratégias, possibilitando a identificação de indivíduos que apresentam um agravo ou doença de interesse de forma a padronizar critérios para o monitoramento das condições de saúde e para a descrição da ocorrência desse evento. O objetivo principal é tornar comparáveis os critérios diagnósticos que regulam a entrada de casos no sistema, tanto no nível nacional quanto internacional(2).

Do ponto de vista da vigilância epidemiológica, a definição de caso pode se modificar ao longo do tempo devido à expansão dos conhecimentos específicos relacionados aos aspectos clínicos e de avaliação complementar, às alterações epidemiológicas e à intenção de ampliar ou reduzir os parâmetros de entrada de casos no sistema, aumentando ou diminuindo sua sensibilidade e especificidade, de acordo com as etapas e as metas estabelecidas por um programa de controle $e^{(1,3)}$.

Como reflexo desse processo dinâmico, a definição de caso de Sífilis Congênita vem passando por diferentes modificações nas últimas duas décadas não apenas no Brasil, mas também em outras partes do Mundo. No caso do Brasil, a Sífilis Congênita tornou-se uma doença de notificação compulsória em 22 de Dezembro de 1986, por meio da Portaria n 542 do Ministério da Saúde (publicada no D.O.U. de 24/12/1986) ${ }^{(4)}$, juntamente com a Síndrome da Imunodeficiência Adquirida (aids). Desde então já houve três ou quatro revisões da definição de caso(5).

A definição de casos de 2004 foi o resultado de reuniões dos Comitês Assessores de Epidemiologia e de Doenças Sexualmente Transmissíveis do Programa Nacional de DST/AIDS realizadas em 2003 e que contaram com a importante participação de representantes da Área Técnica de Saúde da Mulher, da Área Técnica de Saúde da Criança e do Departamento da Atenção Básica, todos do Ministério da Saúde. Além disso, estavam presentes a Federação Brasileira das Sociedades de Ginecologia e Obstetrícia (FEBRASGO) e a Sociedade Brasileira de Doenças Sexualmente Transmissíveis (DST).

O objetivo principal dessa modificação na definição de casos foi o de ajustar a vigilância epidemiológica da sífilis congênita a questões operacionais do sistema de vigilância, mantendo-se a sensibilidade do critério e aumentando a sua especificidade.

A notificação é feita pelo preenchimento e envio da Ficha de Notificação e Investigação Epidemiológica de Caso de Sífilis Congênita, e deve ser preenchida por profissionais de saúde no exercício de sua função. Os dados obtidos são inseridos na base de dados do Sistema de Informação de Agravos de Notificação (SINAN).

A investigação de sífilis congênita será desencadeada nas seguintes situações: 1) todas as crianças nascidas de mãe com sífilis (evidência clínica e/ou laboratorial), diagnosticadas durante a gestação, parto ou puerpério; 2) todo indivíduo com menos de 13 anos de idade com suspeita clínica e/ou epidemiológica de sífilis congênita.

Quatro critérios específicos compõem a definição de caso:

Primeiro Critério: Toda criança, ou aborto (toda perda gestacional, até 22 semanas de gestação ou com peso menor ou igual a 500 gramas), ou natimorto (todo feto morto, após 22 semanas de gestação ou com peso maior que 500 gramas) de mãe com evidência clínica para sífilis e/ou com sorologia não treponêmica reagente para sífilis com qualquer titulação, na ausência de teste 
confirmatório treponêmico, realizada no pré-natal ou no momento do parto ou curetagem, que não tenha sido tratada ou tenha recebido tratamento inadequado.

Considera-se como tratamento inadequado para a gestante todo tratamento feito com qualquer medicamento que não a penicilina; ou tratamento incompleto, mesmo tendo sido feito com penicilina; ou tratamento não adequado para a fase clínica da doença; ou a instituição do tratamento com menos de 30 dias antes do parto; ou elevação dos títulos após o tratamento, no seguimento. Em relação ao(s) parceiro(s), inclui-se como tratamento inadequado para a gestante aquele(s) que não foi(ram) tratado(s) ou foi(ram) tratado(s) inadequadamente segundo as diretrizes de tratamento vigentes, ou quando não se tem essa informação disponível; ou ausência de documentação do tratamento ou da queda dos títulos após tratamento.

Segundo Critério: Todo indivíduo com menos de 13 anos de idade com as seguintes evidências sorológicas: titulações ascendentes (testes não treponêmicos); e/ou testes não treponêmicos reagentes após seis meses de idade (exceto em situação de seguimento terapêutico); e/ou testes treponêmicos reagentes após 18 meses de idade; e/ou títulos

\section{REFERÊNCIAS}

1. Buehler JH. Surveillance. In: Rothman KJ, Greenland S. Modern Epidemiology. $2^{\text {nd }}$ ed. Philadelphia (USA): Lippincot-Raven; 1998. p. 435-57.

2. Waldman EA. Vigilância em Saúde Pública. Vol. 7. São Paulo (SP): Faculdade de Saúde Pública da Universidade Federal de São Paulo Instituto para o Desenvolvimento de Saúde; Núcleo de Assistência Médico-Hospitalar; 1998. Série "Saúde e Cidadania".

3. Laguardia J, Penna ML. Definição de caso e vigilância epidemiológica. Informe Epidemiológico do SUS 1999; 8: 63-6.

4. Brasil. Portaria n 542 de 22 de Dezembro de 1986. Diário Oficial da República Federativa do Brasil, Brasília, 24 de Dezembro de 1986 Seção 1., p. 19827. em teste não treponêmico maiores do que os da mãe.

Em caso de evidência sorológica apenas, deve ser afastada a possibilidade de sífilis adquirida.

Terceiro Critério: Todo indivíduo com menos de 13 anos de idade, com teste não treponêmico reagente e evidência clínica ou liquórica ou radiológica de sífilis congênita.

Quarto Critério: Toda situação de evidência de infecção pelo Treponema pallidum em placenta ou cordão umbilical e/ou amostra da lesão, biópsia ou necropsia de criança, aborto ou natimorto.

Frente aos grandes desafios que a sífilis congênita ainda impõe como problema de saúde pública ${ }^{(6-8)}$, a vigilância epidemiológica deve ser continuamente fortalecida ${ }^{(9)}$. A nova definição de caso de sífilis congênita representa 0 amadurecimento das ações de vigilância frente à realidade e a um contexto epidemiológico e operacional brasileiro. Além disso, deve ser encarada como um estímulo à necessária aproximação entre vigilância e assistência representando uma importante estratégia, tanto para o reconhecimento de sua relevância bem como para o avanço das ações de intervenção e de controle da sífilis congênita no Brasil.

5. Tayra AP. Sistema de vigilância epidemiológica da Sífilis Congênita no Estado de São Paulo [dissertação]. São Paulo (SP): Faculdade de Saúde Pública, Universidade de São Paulo; 2001.

6. Walker DG, Walker GJ. Prevention of congenital syphilis - time for action. Bull World Health Organiz 2004; 82(6):401.

7. Hook EW, Peeling RW. Syphilis control - a continuing challenge. Lancet 2004; 351(2): 122-4.

8. Gutman LT. Syphilis. In: Feigin R, Cherry JD. Textbook of pediatric infectious diseases. $4^{\text {th }}$ ed. Philadelphia (USA): WB Saunders; 1998. p. 1543-56.

9. Centers for Disease Control and Prevention (CDC). Sexually Transmitted Diseases - Treatment Guidelines. Morbidity and Mortality Weekly Report 2002; 51 (RR6): 1-80. 